\title{
Nonlocality and local causality in the Schrödinger Equation with time-dependent boundary conditions
}

\author{
A. Matzkin \\ Laboratoire de Physique Théorique et Modélisation (CNRS Unité 8089), \\ Université de Cergy-Pontoise, 95302 Cergy-Pontoise cedex, France \\ S. V. Mousavi \\ Department of Physics, University of Qom, \\ Ghadir Blvd., Qom 371614-6611, Iran
}

M. Waegell

Institute for Quantum Studies, Chapman University, Orange, CA 92866, USA

\begin{abstract}
We investigate the nonlocal dynamics of a single particle placed in an infinite well with moving walls. It is shown that in this situation, the Schrödinger equation (SE) violates local causality by causing instantaneous changes in the probability current everywhere inside the well. This violation is formalized by designing a gedanken faster-than-light communication device which uses an ensemble of long narrow cavities and weak measurements to resolve the weak value of the momentum far away from the movable wall. Our system is free from the usual features causing nonphysical violations of local causality when using the (nonrelativistic) SE, such as instantaneous changes in potentials or states involving arbitraily high energies or velocities. We explore in detail several possible artifacts that could account for the failure of the SE to respect local causality for systems involving time-dependent boundary conditions.
\end{abstract}




\section{INTRODUCTION}

Nonlocality is the hallmark of quantum mechanics. It is generally taken for granted that nonlocality requires two or more particles, along the lines of the early paper by Einstein Podolsky and Rosen [1], subsequently put into a firm footing by Bell [2]. Although it has been suggested that a single particle could in some instances exhibit nonlocality, such results have been disputed. This is particularly the case of the two main candidates for single particle nonlocality, the Aharonov-Bohm effect [3] and the entanglement between spatial modes of a single photon (see [4] and Refs. therein for previous works), discussed respectively in Refs. [5, 6] and [7, 10].

The present work introduces a new "candidate" for single particle nonlocality. It is based on the fact that the Schrödinger equation solved on a domain with moving boundaries gives rise to apparent violations of local causality. It appears that time-dependent boundary conditions can potentially induce a nonlocal change in a region located far from the location of the moving boundary. Here we will examine the case of a particle in a box with infinitely high but moving walls. We will see that for quantum states extended all over the box, the moving walls generate instantaneously a current density almost everywhere in the box. We will indicate how this effect could be in principle tested, namely by making weak measurements of the particle momentum in the central region of the box before light has the time to propagate from the walls to that region. To this effect, a gedanken faster-than-light communication device will be presented.

Let us state right away that we are not advocating the position that it is possible to send a signal faster than the speed of light. Nevertheless, the present problem is interesting because the non-relativistic Schrödinger equation fails to prevent superluminal signaling in a situation where relativistic considerations do not seem to play a significant role. It is indeed well-known that the Schrödinger equation does not bound particle velocities, nor does it constrain instantaneous changes in potentials, but we will argue that in our system the nonlocal aspects do not rely on spurious violations of special relativity allowed by a employing a nonrelativistic framework.

Note that the effect reported in this work is not due to a non-dynamical phase term, such as a geometric phase (in which case we would have in the present context a nonadiabatic, non cyclic geometric phase [8, 9]). There have been in the past claims that such 
non-dynamical phases in the same type of system that we will be investigating in this work could be envisaged as a specific form of "hidden" (i.e., non-signaling) non-locality [11-13]. We will see instead that the non-local aspect in our candidate system is not based on the existence of such phases.

We will start by revisiting the treatment of systems with time-dependent boundary conditions of the form $\psi(x(t), t)=0$, where $\psi$ is the wavefunction. Such systems are delicate to handle because from a formal point of view a different Hilbert space needs to be defined for each time $t$, so that a simple operation like taking the time derivative $\partial_{t} \psi$ is not straightforward. We will introduce the system we will deal with - a particle in an expanding infinite well - in the context of recent works [14-16] involving time dependent boundary conditions in Sec. 2.

Weak measurements were originally [17] introduced to measure an observable without significantly disturbing the system, allowing a subsequent standard (projective) measurement of a different observable. The outcome, known as a weak value, is not generally an eigenvalue (since the quantum state of the system is barely modified and no projection takes place) but still gives some information on the weakly measured observable, provided enough statistics are gathered by repeating the experience a certain number of times. In particular, it was shown [18] that the weak value of the momentum is directly related to the current density. We will recall these facts in Sec. III where we will present our main results concerning the instantaneous response of the current density to a change in the boundary conditions.

We will then proceed (Sec. 4) to analyze and discuss this novel type of nonlocality. The first issue we will address is no-signaling. No-signaling stands as the major constraint permitting the "peaceful coexistence" [19] of relativity and quantum mechanics. At first sight it would appear that no-signaling is respected here, since a single weak measurement does not convey any information, but the situation is more involved, and a protocol that would allow us to test in principle the possibility of signaling will be presented. Given that this nonlocal effect appears to conflict with the no-signaling principle, we will critically assess the origins of nonlocality, in search of possible artifacts. We will then discuss the present results in the framework of the Bohmian model, where nonlocality is a built-in feature claimed to hold for individual events but is washed out at the statistical level. A summary and our conclusions will be given in Sec. $\mathrm{V}$. 


\section{A PARTICLE IN AN INFINITE WELL WITH MOVING WALLS}

The particle in an infinite well with moving walls was widely investigated in the context of quantum chaos (see e.g. [20-23]). Another line of studies concerning this system involves the conjecture of nonlocality induced by the moving wall on a localized state [12, 24 29], that was recently disproved [16]. The Hamiltonian for a particle of mass $m$ in an infinite well with the left wall fixed at $x=0$ and the right wall moving according to the function $L(t)$ is given by

$$
\begin{aligned}
H & =\frac{P^{2}}{2 m}+V \\
V(x) & =\left\{\begin{array}{l}
0 \text { for } 0 \leq x \leq L(t) \\
+\infty \text { otherwise. }
\end{array}\right.
\end{aligned}
$$

The solutions of the Schrödinger equation $i \hbar \partial_{t} \psi(x, t)=H \psi(x, t)$ must obey the boundary conditions $\psi(0, t)=\psi(L(t), t)=0$. The instantaneous eigenstates of $H$,

$$
\phi_{n}(x, t)=\sqrt{2 / L(t)} \sin [n \pi x / L(t)]
$$

verify $H\left|\phi_{n}\right\rangle=E_{n}(t)\left|\phi_{n}\right\rangle$ where $E_{n}(t)=n^{2} \hbar^{2} \pi^{2} / 2 m L^{2}(t)$ are the instantaneous eigenvalues, but, due to the time varying boundary conditions, the $\phi_{n}$ are not solutions of the Schrödinger equation. To solve the Schrödinger equation different approaches have been proposed, like introducing a covariant time derivative [30, implementing an ad-hoc change of variables [31], or relying on a time-dependent quantum canonical transformation [14, 32]. Here we follow the latter option, as implemented in Ref. [16]. However, rather than going through the transformation to derive the solutions for the general case (this is done in [16]), we will choose from the beginning a specific function $L(t)$ for which analytic basis solutions of the Schrödinger equation are known. Indeed, for the linearly expanding case

$$
L(t)=L_{0}+q t
$$

it can be checked by inspection [31] that

$$
\psi_{n}(x, t)=\sqrt{\frac{2}{L_{0}+q t}} \exp \left(-\frac{i \pi^{2} \hbar^{2} n^{2} t-i L_{0} m^{2} q x^{2}}{2 \hbar m L_{0}\left(L_{0}+q t\right)}\right) \sin \left(\frac{n \pi x}{L_{0}+q t}\right)
$$

verifies the Schrödinger equation and the boundary conditions $\psi(0, t)=\psi(L(t), t)=0$. Here, $q>0$ represents the velocity of the expanding wall. 
The set of $\psi_{n}(x, t)$ (with $n$ a positive integer) form a set of orthogonal basis functions useful to determine the time evolution of an initial arbitrary quantum state. The simplest initial state would be to pick a given $\psi_{n}(x, t=0)$; its evolution follows directly from Eq. (5). From a physical standpoint, it would be more realistic to start from the standard fixed wall eigenfunctions. A typical initial state woud then be an eigenstate $\phi_{n}(x, t=0)$ [see Eq. (3)] or a linear combination thereof, say

$$
\psi(x, t=0)=\sum_{n=1}^{\infty} c_{n} \phi_{n}(x, t=0)
$$

whose evolution is given by

$$
\psi(x, t)=\sum_{k, n} c_{n}\left\langle\psi_{k}(t=0) \mid \phi_{n}(t=0)\right\rangle \psi_{k}(x, t) .
$$

We may want to include additional refinements, like allowing for a continuous transition from the fixed walls to the linear regime by setting

$$
L(t)=L_{0}+q t\left(1-e^{-\gamma t}\right) .
$$

This requires numerical solutions. The numerical method that will be used here is very similar to the one exposed in Ref. [22]; it is based on looking for numerical solutions $\zeta(x, t)$ by using expansions over the instantaneous eigenstates of the form

$$
\zeta(x, t)=\sum_{k=1}^{\infty} a_{k}(t) \phi_{k}(x, t) .
$$

The coefficients $a_{k}(t)$ are retrieved by solving a system (arising by plugging $\zeta(x, t)$ in the Schrödinger equation) of coupled differential equations.

\section{CURRENT DENSITY AND MOMENTUM WEAK VALUES}

\section{A. Current density evolution}

We first briefly look at the standard current density

$$
j=\frac{1}{2 m}\left(\psi^{*} P \psi-\psi P \psi^{*}\right),
$$

where $P$ is the momentum operator for the states in an expanding infinite well. When the initial state is taken to be an eigenstate $\phi_{n}(x, 0)$ of the fixed walls well, given by Eq. 
(3) with all the $c_{k}$ vanishing except for $c_{n}=1$, the current density is initially zero, but becomes non-zero for $t>0$. Indeed, near the wall, the initial wavefunction is nonzero, since $\phi_{n}\left(x \approx L_{0}, t=0\right) \simeq n\left(L_{0}-x\right)$, and is substantially modified when the wall moves. By the arguments given in [16] (or simply by the continuity of the logarithmic derivative noting that the potential remains unchanged except at $x=L(t))$ we then know that at an infinitesimal time $t=\varepsilon$ we will have $\psi_{n}(x, \varepsilon)-\psi_{n}(x, 0) \neq 0$ at any $x$, although we expect this quantity to be large near $x=L(\varepsilon)$ and smaller in the regions away from the moving wall.

When the initial state is taken to be a basis state $\psi_{n}$ given by Eq. (5), the current density is immediately computed as

$$
j_{\psi_{n}}(x, t)=\frac{2 q x \sin ^{2} \frac{n \pi x}{L(t)}}{L(t)^{2}}
$$

We see that $j_{\psi_{n}}(x, t)$ changes continuously both in the space and time variables. The change in the current density at $x \ll L_{0}$ can easily be computed. From

$$
\Delta j(x) \equiv j(x, \varepsilon)-j(x, 0)
$$

we have

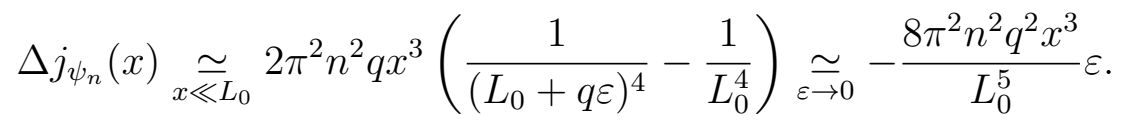

Let $t_{S}=\left(L_{0}-x\right) / c$ be the time it takes for a light signal emitted at the wall to reach the point $x$ where the current density is monitored ( $c$ is the light velocity). Then there is a range

of times $\varepsilon$ such that $\varepsilon<t_{S}$ and $\Delta j(x) \neq 0$ : the current density is modified instantaneously by the wall's motion. The significance of this instantaneous appearance of a current density will be discussed in Sec. IV. We next examine how this current density could in principle be experimentally tested.

\section{B. Weak measurements and the current density}

The underlying idea at the basis of the weak measurement (WM) framework [17] is to give an answer to the question: "what is the value of a property (represented by an observable A) of a quantum system while it is evolving from an initial state $\left|\psi\left(t_{i}\right)\right\rangle$ to a final state $\left|b_{f}\left(t_{f}\right)\right\rangle$ ?". This is done by coupling the system observable $A$ to a dynamical variable of an external pointer, say $Q$ through an interaction Hamiltonian of the form

$$
H_{\text {int }}=g(t) A Q
$$


where $g(t)$ is a smooth function nonzero during the interaction time. The effective coupling constant $g \equiv \int g(t) d t$ is chosen to be very small so that although the system and the external pointer become entangled, the system state is minimally disturbed by $H_{\text {int }}$. A standard measurement of another system observable, say $B$ can then be undertaken. Assume that the eigenvalue $b_{f}$ corresponding to the eigenstate $\left|b_{f}\right\rangle$ is obtained - a step called postselection. It can then be shown ([17]; see e.g. Sec. II of [33] for a brief derivation) that the external pointer that was coupled to $A$ has shifted by the quantity $g A^{w}$ where

$$
A^{w}=\frac{\left\langle b_{f}|A| \psi\left(t_{i}\right)\right\rangle}{\left\langle b_{f} \mid \psi\left(t_{i}\right)\right\rangle}
$$

is known as the weak value of $A$ given the initial (preselected) state $\left|\psi\left(t_{i}\right)\right\rangle$ and the final (postselected) state $\left|b_{f}\right\rangle .{ }^{1}$ Note that while $A^{w}$ is generally a complex quantity, when one weakly measures observable $A$, the shift of the external pointer is proportional to the real part of $A^{w} .{ }^{2}$

Let us now specialize Eq. (15) to a weak measurement of the momentum $P$ immediately followed by a standard measurement of the position, denoting the outcome by $x$. The weak value is then given by $P^{w}=\frac{\langle x|P| \psi\rangle}{\langle x \mid \psi\rangle}$. It is easy to see that $P^{w}$ can be written as [18, 34, 35]

$$
P^{w}=\frac{m j_{\psi}(x, t)}{|\psi(x, t)|^{2}}-i \hbar \frac{\partial_{x}\left(|\psi(x, t)|^{2}\right)}{2|\psi(x, t)|^{2}}
$$

Hence the real part of the momentum weak value is the hydrodynamic velocity (well known from the Bohmian model, see Sec. IV D below) $v(x, t)$ given by

$$
v(x, t) \equiv \frac{j_{\psi}(x, t)}{|\psi(x, t)|^{2}}=\frac{\operatorname{Re} P^{w}}{m} .
$$

Our statement made above on the superluminal change in the current density following the walls' motion has now been couched in terms of an experimentally measurable quantity, the momentum weak value.

${ }^{1}$ For simplicity we have disregarded in Eq. 15 the evolution of the system between the initial preparation time $t_{i}$, the mean interaction time $t_{w}$ and the postselection time $t_{f}$; otherwise Eq. (15) should be replaced by

$$
A^{w}=\frac{\left\langle b_{f}\left|U\left(t_{f}, t_{w}\right) A U\left(t_{w}, t_{i}\right)\right| \psi\left(t_{i}\right)\right\rangle}{\left\langle b_{f}\left|U\left(t_{f}, t_{i}\right)\right| \psi\left(t_{i}\right)\right\rangle}
$$

(see e.g. Sec. II of [33]).

2 The imaginary part of $A^{w}$ is proportional to the shift of the momentum of the pointer wavefunction for a pointer in position space. 
A couple of illustrations are provided in Figs. 1 and 2, where $\operatorname{Re} P^{w}$ is shown as a function of time. Fig. 1 shows the case of a moving wall when the system is initially prepared in a given eigenstate $\phi_{n}$ of the cavity at $t=0$. Fig 2 shows instead the evolution of $\operatorname{Re} P^{w}$ in a static cavity when the system is initially prepared in a basis state $\psi_{n}(x, t=0)$ [Eq. (5)]. In the former case as noted above we will have a nonzero current density (whereas $j(x, t)=0$ for any $t$ if the walls had remained fixed). We see indeed in Fig. 1 that $\operatorname{Re} P^{w}$ changes before a light signal reaches the point where the weak measurement takes place; the light cone boundary $t_{c}=\left(L_{0}-x\right) / c$ is indicated by the red-gridded plane. This is the signature of a form of nonlocality induced by the walls' motion.

In the latter case Eqs. (11) and (17) imply that if the initial state is $\psi_{n}(x, t=0)$, then in a moving cavity the weak value should evolve following

$$
\operatorname{Re} P^{w}(x, t)=\frac{m q x}{L(t)}
$$

This is represented in Fig. 2 by the solid black line. In a fixed cavity instead $\operatorname{Re} P^{w}$ will wildly oscillate, as shown by the blue curves in Fig. 2. Here again the behavior of a distant wall (remaining static or in motion) affects the weak value of the momentum instantaneously, i.e. before a light signal emanating from the wall reaches the point where the weak measurement is made (the light cone boundary appears as the vertical dotted line in Fig. 2).

\section{Weak Measurement Protocol}

We now introduce the type of protocol that could in principle lead to the measurement of $\operatorname{Re} P^{w}$. An infinite square well is realized by a long and narrow cavity. The particle (say an electron) is prepared in an initial state (a given eigenstate of the cavity at $t=0$ or a given basis function, as in Figs. 1 and 2 respectively).

At time $t=0$, the sender Alice is located at $x=L_{0}$ and chooses whether to set the wall in motion (indicating a message bit 1), or to leave the wall at rest (indicating message bit $0)$. Then, a very short time later at $t_{1}$, Bob, located on the opposite side of the cavity near the wall at $x=0$ performs a weak measurement of the position of the particle at location $x=x_{w}$, followed by a strong measurement of the position at $x_{f}$ (in the immediate vicinity of $x_{w}$ ) of the particle at $t_{f}$, again a very short time later. This procedure is equivalent to

measuring the weak value of the momentum. Indeed, it can be shown (see Appendix) that 


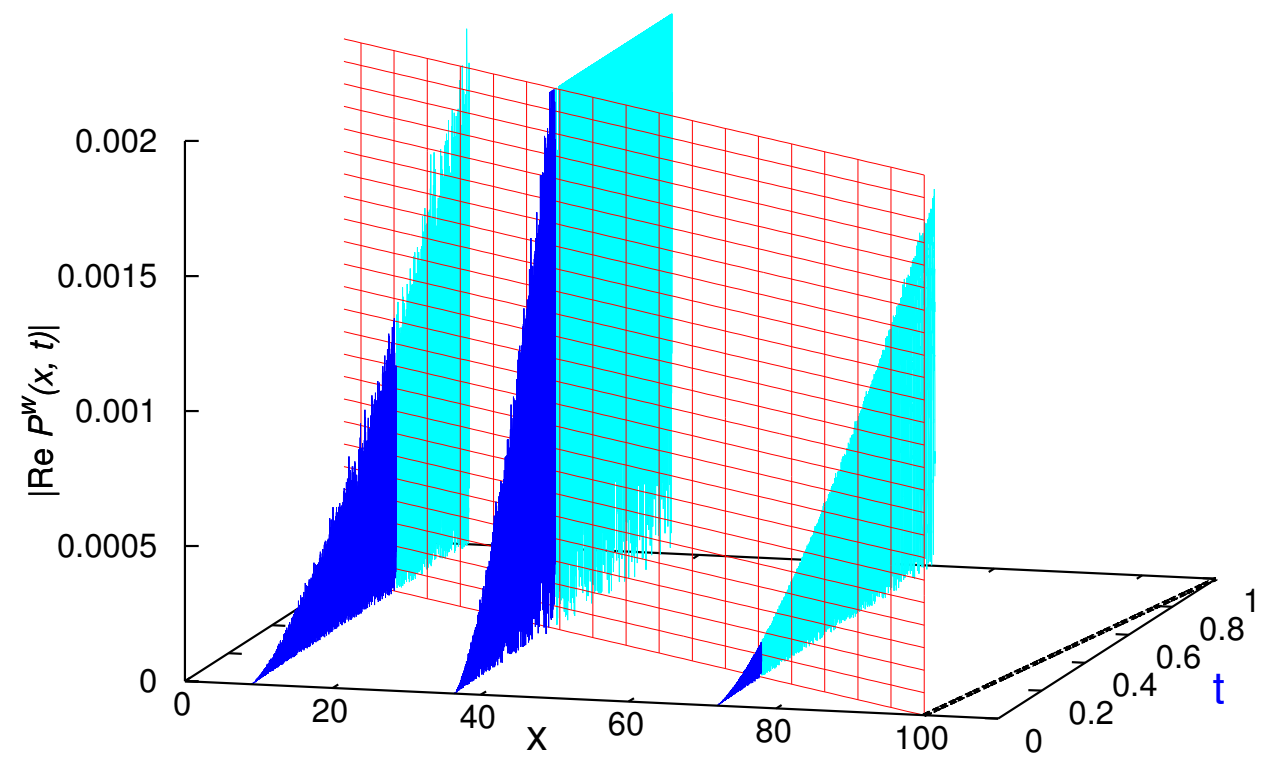

FIG. 1: Time evolution of the weak value of the momentum of an electron in an expanding cavity of initial length $L_{0}=100 \mathrm{au}$. The blue curves represent $\left|\operatorname{Re} P^{w}(x, t)\right|$ (where $P^{w}$ is the momentum weak value, see Eq. (17) obtained by making a weak measurement at the corresponding value of $x$. The wall is initially at $L_{0}$ and the red-gridded plane represents the boundary of the light cone originating from $x=L_{0}$ at $t=0$. Initially $\operatorname{Re} P^{w}(x, t=0)=0$ everywhere (and would stay as such for a static wall) but is seen to oscillate before the light cone reaches the points were $P^{w}$ is determined (the light blue curves represent $P^{w}$ for times inside the light cone). The dashed line to the right schematically represents the walls motion given by $L(t)$. The following parameters have been used: $q=0.5, m=1$, initial wavefunction chosen to be an eigenstate of the static well $\phi_{n}(x, 0)$ [see Eq. (3)] with $n=11$ (numbers given in atomic units (au)).

with the weak value of the position given by

$$
X^{w}=\frac{\left\langle x_{f}\left|U\left(t_{f}, t_{w}\right) X\right| \psi\left(t_{w}\right)\right\rangle}{\left\langle x_{f} \mid \psi\left(t_{f}\right)\right\rangle}
$$

where $U\left(t_{f}, t_{w}\right)$ is the evolution operator between $t_{w}$ and $t_{f}$, we have

$$
P^{w}=\lim _{t_{f} \rightarrow t_{w}} \frac{m}{t_{f}-t_{w}}\left(x_{f}-X^{w}\right) .
$$




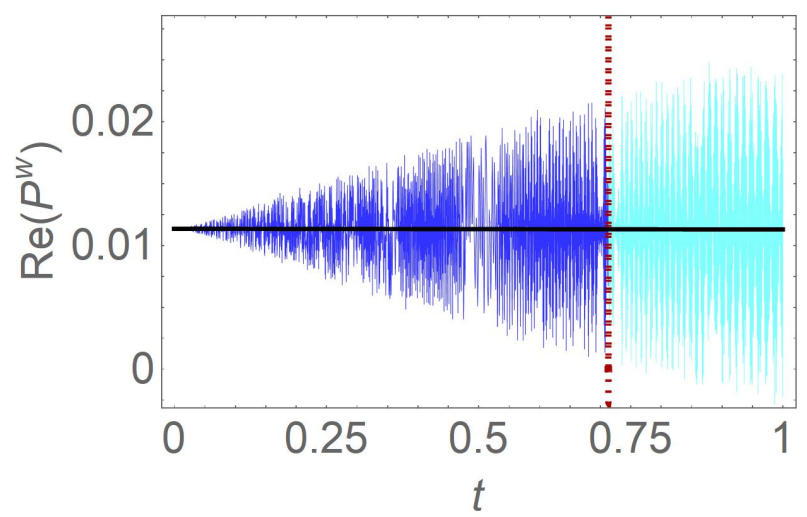

FIG. 2: Time evolution of the weak value $\operatorname{Re} P^{w}\left(x_{f}, t\right)$ of the momentum for $x_{f}$ lying near the origin (i) in an expanding cavity (solid black line) (ii) in a static cavity (blue and light blue lines). The red-dashed line is the border of the light cone (the values of $\operatorname{Re} P^{w}\left(x_{f}, t\right)$ inside the light cone are in light blue). The fact that the wall moves or remains fixed is instantaneously reflected in the behavior of the weak value. The parameters used are $L_{0}=100, x_{f}=2.27, m=1$, initial wavefunction chosen to be a basis function $\psi_{n}(x, 0)$ [see Eq. (5)] with $n=44$ and in the moving case $q=0.5$ (numbers given in atomic units $(\mathrm{au})$ ).

Note that the protocol can also be implemented with a direct weak measurement of the momentum, rather than the two position measurements leading to Eq. 21). This type of weak measurement of the momentum relies on a particular coupling between the particle momentum and an external pointer (in practice, the external pointer is often another degree of freedom of the particle). The important point is that Bob carries out the weak measurement procedure before a light signal sent by Alice at $t=0$ reaches him, that is we must have $t_{f}<L_{0} / c$.

Bob can measure whether Alice sent a bit 0 or a bit 1 . When the initial state is a stationary state $\phi_{n}(x, 0)$ of the cavity $P^{w}=0$ if the wall remains fixed, but takes a nonzero value (as in Fig. 1) if the wall was set in motion. When the intial state is a basis state $\psi_{n}(x, 0)$, $\operatorname{Re} P^{w}$ is given by Eq. (19) if the wall moves, so Bob can verify by making successive weak measurements on the system if $\operatorname{Re} P^{w}$ is consistent or departs from Eq. (19), as displayed in Fig. 2,

Nevertheless, weak measurements are noisy, and it is impossible for Bob to learn Alice's choice of signal bit in a single run of the experiment. First the postselection probability 
$\int_{x_{w}-\epsilon}^{x_{w}+\epsilon}\left|\psi\left(x, t_{f}\right)\right|^{2} d x$ is very small ( $\epsilon$ is the width over which the weak measurement takes place). Second, by definition, when weakly measuring observable $X$, the pointer wavefunction incurs small shifts [see Eq. (14)] relative to its width, so many runs of the same experiment will be necessary in order to extract the weak values. Third even in a weak measurement there is inevitably a back action of the coupling interaction on the subsequent evolution leading to the post-selection. The universal part of the back action is encoded in the imaginary part of the weak value [36]. A large imaginary part will distort the external pointer state and make even more difficult to extract the small shift of the pointer wavefunction. We note here that for an initial state of the form given by Eq. (5), for which we computed $\operatorname{Re} P^{w}(x, t)=m q x / L(t)[$ Eq. (19)], we have

$$
\operatorname{Im} P^{w}=-\frac{\hbar \pi n}{L(t)} \cot \left(\frac{n \pi x}{L(t)}\right) .
$$

The real and imaginary parts of $P^{w}$ follow a different behavior, and Eq. 22 gives an indication of initial states and spatial regions minimizing the back action.

However, unlike many other cases where quantum uncertainty prevents superluminal signaling, we will see that this setup has no such limitation. This is a serious problem that calls for a more detailed discussion of non-locality and no-signaling, and compels us to explore possible artifacts of the model.

\section{DISCUSSION}

\section{A. Relativity and non-locality}

The difficulties in reconciling quantum mechanics and special relativity are well-known [37]. These difficulties stem from the global character of the state vector, defined in a mathematical configuration space and not in physical space. It is generally accepted that quantum correlations cannot lead to superluminal communication of information (no-signaling) as this would indeed result in an open conflict with relativity. Instead, a "peaceful coexistence" [19] between quantum mechanics and relativistic constraints is advocated: as long as one does not attempt to understand how the quantum correlations come about (in particular through a hidden-variable model or by endowing the state vector with physical reality), the observed statistics predicted by quantum theory respect no-signaling. However if the state vector is 


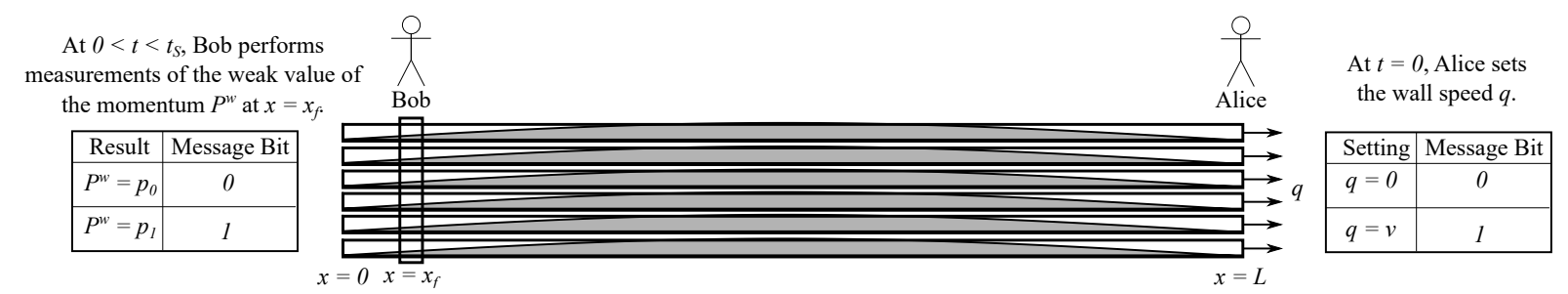

FIG. 3: A gedanken experiment enabling faster-than-light communication using a large ensemble of long narrow cavities, each containing a single particle prepared in the same state $\Psi$ (none of the particles are entangled). Alice sends her message at $t=0$ and Bob receives the message at $0<t<t_{S}$, where $t_{S}$ is the time for a light signal sent by Alice at $t=0$ to reach Bob, thus violating the no-signaling principle.

assumed to be linked to a real process, then individual events are difficult to reconcile with relativistic invariance. This is the case for the collapse of the state vector upon measurement [38], or for sub-quantum theories such as the Bohmian model (see Sec. IVD below).

The apparent non-locality seen in the infinite well with a moving wall investigated here conflicts with this view. The reason is that the non-local effect comes about as the direct result of a change in a single-particle state vector (rather than a multi-particle entangled state). We will first modify the weak measurement protocol given above in Sec. IIIC to show how it can lead to signaling. We will then discuss the possible artifacts that could explain our results.

\section{B. Bypassing no-signaling with weak measurements}

Again, the noisy nature of the weak measurements in the protocol of Sec. IIIC does not enable discrimination between the cases of a moving wall and fixed wall in a single run. However, since only Bob makes the weak measurement and the strong postselection measurement, we can couch the statistical argument involving many runs in terms of a single experiment involving many copies of the system, as shown in Fig. 3 ,

Consider a gedanken experiment taking the form of a very large ensemble of extremely long and narrow cavities, all aligned together, each with a particle prepared in the same suitably-chosen state $\Psi$ of the cavity. At time $t=0$, the sender Alice, located at $x=L_{0}$ chooses either to set all of those walls in motion (indicating a message bit 1), or to leave them 
all at rest (indicating message bit 0). Then, as in Sec. III C, Bob, located on the opposite side of the cavity near the wall at $x=0$ performs on each cavity a weak measurement of the position of the particle at location $x=x_{w}$, followed a very short time later by a strong measurement of the position at $x_{f}$ of the particle at $t_{f}$.

Next, Bob considers the weak measurement data for the sub-ensemble of cavities wherein postselection (detection of the particle at $x_{f}$ ) was successful. The data allows him to infer the real part of the weak value $P^{w}$ at $x_{w}$. If the walls are moving, Bob detects the weak momentum $p_{1}$, indicating a message bit of 1 , and otherwise Bob detects the weak momentum $p_{0}$, indicating a message bit of 0 . The values of $p_{0}$ and $p_{1}$ depend on the initial state $\Psi$ in the cavities, which is ideally chosen to make them easily distinguishable, as with $\phi_{n}$ (Fig. 1 ) and $\psi_{n}$ (Fig. 2).

In principle we can make the ensemble arbitrarily large, and the cavities arbitrarily long,

allowing Bob to have enough time to collect sufficient weak measurement data before a light signal sent from Alice at $L_{0}$ reaches him. Hence this device enables Alice to send a signal to Bob faster than the speed of light. All of this analysis is built upon the basis solutions $\psi_{n}(x, t)$, and thus it appears that these solutions must be nonphysical if local causality is to be respected. In the following sections we discuss possible reasons that these solutions are flawed.

\section{Sources of nonlocality and possible artifacts}

The most obvious candidates to account for the apparent nonlocality examined in this work would be the action at a distance effects allowed by a nonrelativistic formalism such as the Schrödinger equation. There are two types of sources that give rise to superluminal features. First, the existence of instantaneous potentials. Second, the fact that a nonrelativistic framework does not place any restriction on the energy (and hence velocity) components of a given wavefunction. We argue why these two features can be discarded in accounting for the results obtained here. We then examine other possible artifacts, including the fact that unlike a wave equation of the d'Alembert type, the Schrödinger equation does not impose a particular wave speed, which enables waves to propagate instantaneously. 


\section{Instantaneous potentials}

The instantaneous propagation of potentials appears at first as irrelevant to our problem: inside the box (except in the vicinity of $x=L(t)$ ), and in particular in the region close to $x_{f}$, the potential remains zero at all times. The potential is therefore not modified so that the question of its instantaneous propagation appears to be moot.

Nevertheless it should be mentioned that formally, the proper way of obtaining the basis solutions (see [16] and Refs. therein) given by Eq. (5) involves a time-dependent unitary transformation mapping the moving boundaries problem to a different system with fixed boundaries. The Hamiltonian of the transformed system is

$$
h(t)=\frac{P^{2} L_{0}^{2}}{2 m L^{2}(t)}-\frac{\partial_{t} L(t)}{2 L(t)}(X P+P X)
$$

with vanishing boundary conditions at both ends of the interval $\left[0, L_{0}\right] . h(t)$ can be understood as describing a system in a fixed wall infinite potential well with a time-dependent mass and subjected to a time and velocity dependent potential. In this mapped system that, contrary to the original problem, is described in a single well-defined Hilbert space the instantaneous and uniform character of the time-dependence is obvious. It is however unlikely that one can make valid inferences concerning the physics of the original system from the physics of the mapped system (for example the issue of signaling does not even arise in $h(t))$. Quite the contrary, the global and time-dependent aspects of the mapped system are readily understood as unphysical features due to the dilation imposed by the unitary transformation on the original system.

\section{Infinite velocities}

The issue of infinite velocities arises because in the nonrelativistic framework a given wavefunction may contain, when expanded over the energy eigenstates, high energy states that can account for faster than light propagation. This artifact, due to the nonrelativistic nature of the Schrödinger equation, has been known to produce apparent superluminal propagation in several instances, in particular when the wavefunction has a discontinuous cut-off. For example in the quantum shutter problem [39], it was shown [40] that a super-

luminal propagation occurs due to the high frequencies needed to account for the cutoff of the initial wavefunction, before the shutter is released. 
In the present problem, the contribution of high energy states can be evaluated by expanding the solution of the Schrödinger equation $\psi(x, t)$ as given by Eq. (7) in the instantaneous eigenstate basis. Let us assume that a cavity is initially in the fixed wall eigenstate $\phi_{n_{0}}(x, t=0)$ [cf. Eq. (3)]. In order to compute the evolution, we need to expand $\phi_{n_{0}}(x, t=0)$ over the basis functions $\psi_{k}(x, t=0)$, as in Eq. (7) but here with a single term $n_{0}$. The overlap coefficients

$$
d_{k n}(t)=\int_{0}^{L(t)} \psi_{k}^{*}(x, t) \phi_{n}(x, t) d x
$$

can be readily computed in closed form as

$$
d_{k n}(t)=\frac{e^{-i \pi / 4} \sqrt{\hbar \pi}}{2 \sqrt{2 M}}\left(\begin{array}{c}
e^{\frac{i \pi^{2} \hbar\left(k^{2} q t+L_{0}(k-n)^{2}\right)}{\left.2 L_{0} M\right)}}\left[\operatorname{erf}\left(\frac{e^{i \pi / 4}(\pi \hbar(k-n)+M)}{\sqrt{2 \hbar M}}\right)+\operatorname{erf}\left(\frac{e^{i \pi / 4}(\pi \hbar(n-k)+M)}{\sqrt{2 \hbar M}}\right)\right] \\
-e^{\frac{i \pi^{2} \hbar\left(k^{2} q t+L_{0}(k+n)^{2}\right)}{\left.2 L_{0} M\right)}}\left[\operatorname{erf}\left(\frac{e^{i \pi / 4}(\pi \hbar(k+n)+M)}{\sqrt{2 \hbar M}}\right)-\operatorname{erf}\left(\frac{e^{i \pi / 4}(\pi \hbar(k+n)-M)}{\sqrt{2 \hbar M}}\right)\right]
\end{array}\right)
$$

with $M \equiv m q L(t)$.

We first need to determine $d_{k n_{0}}(t=0)$. Assuming $n_{0}$ is small, each square bracket in Eq. 255 is seen to vanish for $k \gg M / \pi \hbar$ when the error functions cancel out, so the infinite sum in Eq. (7) can actually be cut off at a value somewhat larger (depending on the mass) than $\bar{k}=M / \pi \hbar$. The contribution of an instantaneous eigenstate $\phi_{n}(x, t)$ at time $t$ is then given by

$$
\left\langle\phi_{n}(t) \mid \psi(t)\right\rangle=\sum_{k=1}^{k_{\text {cut }}} d_{k n_{0}}(0) d_{k n}^{*}(t)
$$

where $k_{\text {cut }}$ is the cutoff value. From Eq. 25) it is seen that the error functions in the brackets will cancel each other for $n \gg \bar{k}$. The modulus of the velocity in an instantaneous eigenstate $\phi_{n}(x, t)$ is given by

$$
v(n, t)=\hbar \pi n / m L(t)
$$

and for $n=\bar{k}$, this becomes $v(\bar{k})=q$. Therefore, depending on the mass, we can expect from the properties of the error function that the highest energy eigenstates that will contribute will have at most a corresponding velocity 2 or 3 orders of magnitude larger than $q$, the velocity at which the wall is expanding, which can be arbitrarily smaller than $c$.

Mathematically the tail remains as $d_{k n}$ falls off as $1 / n^{3}$ for large $n$ and is not strictly zero. The physical effects relevant to the tail can generally be ignored, at least as quantities related directly to the wavefunction are concerned (for instance when comparing the wavefunction 
evolution $\psi(x, t)-\psi(x, 0))$. This can be confirmed numerically. Let us consider an electron in a cavity with the parameters given in Fig. 1. To quantify the tails, we note that

$$
\sum_{k=1}^{k_{\text {cut }}}\left|d_{k n_{0}=11}(0)\right|^{2}=1
$$

holds with $k_{\text {cut }}=650$ if the numerical zero is set at $10^{-10}$ (meaning that the states with $k>650$ account for a relative part of less than $10^{-10}$ in the total state vector). At some arbitrary time $t$, the solution $\psi(x, t)$ is expanded over the instantaneous eigenstates (3). Now

$$
\sum_{n=1}^{n_{\mathrm{cut}}}\left|\sum_{k=1}^{k_{\mathrm{cut}}} d_{k n_{0}=11}(0) d_{n k}^{*}(t)\right|^{2}=1
$$

holds again up to $10^{-10}$ for $n_{\text {cut }}=650$ for, say $t=t_{S} / 2$ (a light signal sent from the right end of the well is halfway through in the fixed wall frame). According to Eq. (27) $v\left(n=650, t_{S}\right) / c<15 \%$. Hence if we neglect the tail, we recover the wavefunction up to one part in $10^{-10}$ while remaining far from the regime of superluminal velocities.

However concerning the current density, the quantities we are looking at are very small and convergence to the same numerical zero taken for the wavefunction is achieved only when including $d_{k n}(t)$ coefficient lying in the tail. For example when the initial state is $\phi_{n_{0}}(x, t=0)$, the current density in the moving cavity is initially zero, and for small $x$ values $j(x, t)$ rises slowly. The determination of $j(x, t)$, which needs to be done by expanding over the basis functions, converges by including expansion coefficients lying in the tail and corresponding to velocities with arbitrarily high energies. To be clear, $j(x, t)$ is nonzero if coefficients in the tail are excluded, but convergence is only achieved when coefficients in the tail are included. In this sense, going into the tail (i.e., including arbitrarily high energies into the computation) appears as a mathematical requirement to obtain a stable result rather than giving rise to the phenomenon itself.

We can therefore conclude that the nonlocal effect put into evidence above does not appear to be due to the existence of arbitrarily high velocities that would propagate the change in the quantum state at an arbitrarily high velocity. The ambiguity in the inclusion of the tail terms (necessary to achieve convergence for the initial states we have worked with here) can only be lifted by working within a fully relativistic framework. 


\section{Model artifact and wave equations}

Mathematically, the source of nonlocality is straightforward to pinpoint. As can be read off from Eqs. (17) or (21), the instantaneous change of the wavefunction at every point of space as time unfolds (due to the expansion of the cavity) is what changes the current density or the momentum weak value. From a formal point of view, the peculiarity of the model, as mentioned in Sec. II, is that at each time $t$, the system is defined on a different Hilbert space. Following the system evolution as time unfolds implies connecting vectors belonging to different Hilbert spaces. Hence, connecting "independent" solutions belonging to different Hilbert spaces might result in an unphysical picture, resulting in fictitious instantaneous effects. Note that such a phenomenon is expected to be ubiquitous when the potential changes in a specific restricted region of space: the state vector changes instantaneously in Hilbert space, leading to a modification of the wavefunction in the regions in which the potential was not modified.

This is related to the fact that from the point of view of wave equations, we know that the Schrödinger equation does not impose a finite propagation velocity. It might be conjectured that by supplementing the Schrödinger equation with a propagation velocity, as is the case of the relativistic Klein-Gordon equation or d'Alembert equation for Maxwell fields, we would get rid of these nonlocal effects. We can pursue the analogy with classical electromagnetism further: from the point of view of Maxwell fields in an expanding cavity, we would see the instantaneous change of standing waves as an artifact of the model. Indeed, we can rely on Maxwell's equations to take into account the transient effects (radiation and propagation) due to the moving charges composing the wall. However in standard unitary quantum mechanics there are no fundamental equations underlying the Schrödinger evolution on which we could rely to take into account this specific transient effect. Note that the basis functions (5) are exact solutions of the Schrödinger equation: they play the same role in the present problem as the Moshinsky function in the paradigmatic shutter problem [39, 41]. The Moshinsky functions form the transient basis solutions of the Schrödinger equation in the shutter problem [41]. It thus looks like taking into account a putative transient phenomenon for our moving wall problem would need to supplement the standard quantum formalism. 


\section{Signaling and the Bohmian model}

As we noted below Eq. (17), the real part of the momentum weak value $P^{w}$ is essentially the velocity of the particle postulated in the de Broglie-Bohm interpretation [42] (in short $\mathrm{dBB}$ or Bohmian model). Recall that dBB accounts for quantum phenomena by postulating the existence of point-like particles guided by the wavefunction. If we write the wavefunction in polar form as

$$
\psi(x, t)=\rho(x, t) \exp (i \sigma(x, t) / \hbar)
$$

then $\rho$ and $\sigma$ obey the equation

$$
\frac{\partial \sigma}{\partial t}+\frac{(\nabla \sigma)^{2}}{2 m}+V+Q=0
$$

where $V$ is the usual potential and the term

$$
Q(x, t) \equiv-\frac{\hbar^{2}}{2 m} \frac{\partial_{x}^{2} \rho}{\rho}
$$

is known as the quantum potential. The particle velocity, defined from within the Bohmian

model by $v(x, t)=\partial_{x} \sigma(x, t) / m$ rather than the equivalent Eq. (18) obeys a Newton law modified by the presence of the quantum potential:

$$
m \frac{d v}{d t}=-\partial_{x}(V+Q)
$$

Bohmian trajectories in systems analogous to the one investigated here have been previously computed [29].

The Bohmian model is generally recognized as being nonlocal. The culprit is the quantum potential, whose local value depends on the instantaneous positions of all particles in the universe. Hence, a Bohmian particle is instantaneously affected by the motion of all of those particles, including those which produce the effective barriers of a potential well. Nevertheless it is generally accepted by proponents of the model that this nonlocality cannot be used to communicate due to the intrinsic quantum randomness (which also includes but is not limited to the ignorance of the particle's initial condition in a given realization). There is therefore nonlocality at the individual level, but because of the random character of quantum mechanics, no-signaling holds at the statistical level.

In the Bohmian account of our device introduced in Sec. III C, the postselected particle at $x_{f}$ was assumed to be there even before it was detected. However its dynamics were affected 
by the quantum potential, which carries the influence of the far wall's motion. Here, the superluminal signaling aspect of our protocol yields a conflict with the usual notion that $\mathrm{dBB}$, despite being explicitly nonlocal, obeys the no-signaling principle. For instance in the EPR-Bell setting involving two particles in an entangled state, the quantum potential changes instantaneously, thereby "contradicting the spirit of relativity" (as put nicely by Holland, cf Sec. 11.3 of [42]), although this change has no observable consequences. In our system, the only quantity that changes instantaneously is the quantum potential given by Eq. (32), since as we remarked above, the usual potential remains constant except in the vicinity of the wall, but there are observable consequences. Note that the enforcement of a finite propagation velocity mentioned in Sec. IV C 3 in dBB would constitute a constraint on the propagation speed of the quantum potential itself.

\section{CONCLUSION}

To summarize, we have put forward a model displaying an apparent single-particle nonlocality and enabling faster-than-light communication. As discussed in Sec. IV, we believe these effects are artifacts of the fact that we are using the nonrelativistic Schrödinger equation, and not genuine physical effects that will one day be realized as actual communication devices. However, even after this analysis, we still cannot claim to have pinned down exactly why the Schrödinger equation violates local causality, even in a regime where it seems relativistic effects should be negligible.

From a physical standpoint it seems likely that there must be a transient behavior which begins at the moving wall and propagates through the wavefunction at or below $c$. These transient behaviors are not given by the solutions of the standard Schrödinger equation, so it seems plausible to suggest that the correct dynamical evolution equation would contain terms accounting for such transients. Even presuming this is the right way to impose a relativistic constraint on the non-relativistic Schrödinger equation, we do not presently have a suggested form for incorporating this constraint. A relativistic treatment would be helpful, despite the well-known limitations affecting the single particle relativistic wavefunctions.

To conclude, the system investigated in this work raises interesting questions about the general trustworthiness of any solutions to the Schrödinger equation involving timedependent potentials localized in a given spatial region but affecting the entire wavefunction. 
We remain open to the possibility that there could be some other explanation that we have not considered, and we would be very pleased if a more complete resolution of this conundrum could be found.

Acknowledgments: AM thanks the participants of a FQXI workshop in Marseille (July 2017) for useful exchanges on single particle nonlocality. MW acknowledges partial support from the Fetzer Franklin Fund of the John E. Fetzer Memorial Trust.

\section{Appendix: Weak value of the momentum in terms of position measurements}

We prove here Eq. 211) expressing $P^{w}$ in terms of a weak and then a projective position measurements. The manipulations are similar to the ones employed in Ref. [34. With $\Delta t \equiv t_{f}-t_{w}$ very small, we have

$$
X^{w}=\frac{\left\langle x_{f}\left|U\left(t_{f}, t_{w}\right) X\right| \psi\left(t_{w}\right)\right\rangle}{\left\langle x_{f} \mid \psi\left(t_{f}\right)\right\rangle}=\frac{\left\langle x_{f}\left|\left(1-\frac{i}{\hbar} \Delta t H\right) X\right| \psi\left(t_{w}\right)\right\rangle}{\left\langle x_{f}\left|\exp \left(-\frac{i}{\hbar} \Delta t H\right)\right| \psi\left(t_{w}\right)\right\rangle} .
$$

Since $i[H, X]=\hbar P$ we have

$$
\begin{aligned}
X^{w} & =\frac{\left\langle x_{f}\left|\left(X-\frac{\Delta t}{m} P-\frac{i \Delta t}{\hbar} X H\right)\right| \psi\left(t_{w}\right)\right\rangle}{\left\langle x_{f}\left|\exp \left(-\frac{i}{\hbar} \Delta t H\right)\right| \psi\left(t_{w}\right)\right\rangle} \\
& =\frac{x_{f}\left\langle x_{f} \mid \psi\left(t_{w}\right)\right\rangle-\frac{\Delta t}{m}\left\langle x_{f}|P| \psi\left(t_{w}\right)\right\rangle-x_{f}\left\langle x_{f}\left|\left(1-\exp \left(-\frac{i}{\hbar} \Delta t H\right)\right)\right| \psi\left(t_{w}\right)\right\rangle}{\left\langle x_{f}\left|\exp \left(-\frac{i}{\hbar} \Delta t H\right)\right| \psi\left(t_{w}\right)\right\rangle} \\
& =\frac{-\frac{\Delta t}{m}\left\langle x_{f}|P| \psi\left(t_{w}\right)\right\rangle}{\left\langle x_{f}\left|\exp \left(-\frac{i}{\hbar} \Delta t H\right)\right| \psi\left(t_{w}\right)\right\rangle}+x_{f} .
\end{aligned}
$$

Therefore

$$
\frac{\left\langle x_{f}|P| \psi\left(t_{w}\right)\right\rangle}{\left\langle x_{f}\left|\exp \left(-\frac{i}{\hbar} \Delta t H\right)\right| \psi\left(t_{w}\right)\right\rangle}=\frac{m}{\Delta t}\left(x_{f}-X^{w}\right)
$$

and taking the limit $\Delta t \rightarrow 0$ (or equivalently $t_{f} \rightarrow t_{w}$ ) we recover Eq. (21).

[1] A. Einstein, B. Podolsky and N. Rosen, Phys. Rev. 47, 777 (1935).

[2] J.S. Bell, Speakable and unspeakable in quantum mechanics (Cambridge University Press, Cambridge, 2004), Chap. 4. [Original publication in Foundations of Quantum Mechanics NewYork:Academic, 1971), p. 171-181].

[3] Y. Aharonov, E. Cohen, and D. Rohrlich, Phys. Rev. A 93, 0421102016 
[4] S.-Y. Lee, J. Park, J. Kim, and C. Noh, Phys. Rev. A 95, 0121342017

[5] L. Vaidman Phys. Rev. A 86, 040101(R) 2012

[6] K. Kang Phys. Rev. A 91, 052116, 2015

[7] G. Björk, P. Jonsson, and L. L. Sánchez-Soto, Phys. Rev. A 64, 0421062001

[8] J. Samuel and R. Bhandari, Phys. Rev. Lett. 60, 23391988.

[9] N. Mukunda and R. Simon, Ann. Phys. 228, 205, 1993.

[10] M. O. Terra Cunha, J. A. Dunningham and V. Vedral, Proc. R. Soc. A 46322772007

[11] Y. Aharonov, Proc. Int. Symp. Found. Quant. Mech. (Tokyo, 1983). Reprinted in S Nakajima, Y Murayama and A Tonomura (Eds), Foundations of Quantum Mechanics in the Light of New Technology (World Scientific, Singapore, 1997), p. 10.

[12] D. M. Greenberger Physica B 151374 (1988)

[13] Y. Aharonov and D. Rohrlich, Quantum Paradoxes: Quantum Theory for the Perplexed (Wiley VCH, Weinheim, 2005), see Sec. 6.1 and 6.2 of Ch. 6.

[14] S. Di Martino, F. Anza, P. Facchi, A. Kossakowski, G. Marmo, A. Messina, B. Militello and S. Pascazio, J. Phys. A 46365301 (2013).

[15] E. Knobloch and R. Krechetnikov, Acta Appl. Math. 137123 (2015) .

[16] A. Matzkin, J. Phys. A 51095303 (2018)

[17] Y. Aharonov, D. Z. Albert, and L. Vaidman, Phys. Rev. Lett. 60, 1351 (1988).

[18] C. R. Leavens Found. Phys. 35469 (2005). ()

[19] A. Shimony, International Philosophical Quarterly, 18, 3 (1978).

[20] P. Seba, Phys. Rev. A 412306 (1990).

[21] C. Scheininger and M. Kleber, Physica D 50391 (1991).

[22] M.L. Glasser, J. Mateo, J. Negro, and L.M. Nieto, Chaos Solitons Fract. 412067 (2009).

[23] V. Grubelnik, M. Logar, and M. Robnik, J. Phys. A 47355103 (2014).

[24] A. J. Makowski and P. Peplowski, Phys. Lett. A 163142 (1992).

[25] J. Zou and B. Shao Int. J. Mod. Phys. B 141059 (2000).

[26] Q.K. Yao Qian-Kai, G. W. Ma, X. F. Chen and Y. Yu Int. J. Theor. Phys. 40551 (2001).

[27] Z. S. Wang, C. Wu, X. L Feng, L.C. Kwek, C.H. Lai, C.H. Oh and V. Vedral Phys. Lett. A 372775 (2008)

[28] S. V. Mousavi, EPL 9930002 (2012).

[29] S. V. Mousavi Phys. Scr. 86035004 (2012); Phys. Scr. 89065003 (2014). 
[30] P. Pereshogin and P. Pronin Phys. Lett. A 1562 (1991)

[31] A.J. Makowski and S.T. Dembinski, Phys. Lett. A 154217 (1991)

[32] A. Mostafazadeh J. Phys. A 328325 (1999)

[33] Q. Duprey and A. Matzkin Phys. Rev. A 95, 032110 (2017)

[34] H. M. Wiseman, New J. Phys. 9, 165 (2007).

[35] A. Matzkin, Phys. Rev. Lett. 109150407 (2012) (Supp. Mat.)

[36] Y. Aharonov and A. Botero, Phys. Rev. A 72, 052111 (2005)

[37] A. Peres and D. Terno, Rev. Mod. Phys. 76, 93 (2004)

[38] Y. Aharonov and D. Z. Albert Phys. Rev. D 29, 228 (1984)

[39] M. Moshinsky Phys. Rev. 84, 525 (1951).

[40] G. Garcia-Calderon, A. Rubio, and J. Villavicencio Phys. Rev. A 59, 1758 (1999)

[41] A. del Campo, G. García-Calderón and J.G. Muga, Phys. Rep. 4761 (2009).

[42] P. Holland, The Quantum Theory of Motion (Cambridge Univ. Press, Cambridge, 1995). 